\title{
Cost Analysis of Surgical Approaches Performed by Residents for Bladder Outlet Obstruction: Laser versus Loop*
}

\author{
Samuel H. Eaton ${ }^{1}$, Courtney K. Rowe ${ }^{2}$, Mark B. Del Papa ${ }^{3}$, Lori B. Lerner ${ }^{3 \#}$ \\ ${ }^{1}$ Northwestern University, Chicago, USA \\ ${ }^{2}$ Harvard Longwood Program, Boston, USA \\ ${ }^{3}$ VA Boston Healthcare System, Boston, USA \\ Email: "Lerner_lori@hotmail.com
}

Received March 16, 2012; revised March 23, 2012; accepted April 20, 2012

\begin{abstract}
Purpose: Few studies have analyzed cost differences between holmium laser enucleation of the prostate (HoLEP) and transurethral resection of the prostate (TURP), and none as regards resident training. We compared these costs at a teaching institution with residents from two Boston programs. Methods: We reviewed all patients who underwent TURP (January 2007-August 2010) or HoLEP (April 2008-August 2010) with residents, excluding those with prostate cancer, simultaneous procedures at the same time, or prior urethral procedures. Operative approach was determined following consultation between the senior resident and the attending surgeon. Operative, postoperative, and urologic follow-up costs were captured and analyzed from day of surgery to 6 months post-operatively. Costs were calculated by the Department of Decision Support Services. Results: 38 HoLEP and 23 TURP patients met inclusion criteria. The two groups were comparable with the exception of higher ASA score and anticoagulation use in the HoLEP group. Despite a decreased hospital stay ( 0.42 vs. 1.25 days), total costs for HoLEP were higher than TURP ( $\$ 8380.00$ vs. $\$ 5861.78, \mathrm{p}<0.05)$ due to higher operative times $(123 \mathrm{~min}$ vs. $74 \mathrm{~min}, \mathrm{p}<0.05)$, resulting in higher operative costs (\$6768.14 vs. \$3853.35, p < 0.05). Conclusions: HoLEP costs are higher than TURP from longer operative times and higher intraoperative costs, partly due to resident teaching. However, senior residents more often selected HoLEP for medically complex and/or anticoagulated patients. Despite resident inexperience with HoLEP, the complication rate remained low. Higher costs must be weighed against HoLEP benefits, which include less morbidity, shorter hospital stays and faster recovery times.
\end{abstract}

Keywords: Holmium; Laser; Benign Prostatic Hyperplasia; Cost; Resident

\section{Introduction}

While results of Holmium Laser Enucleation of the Prostate (HoLEP) have been shown to be equivalent or superior to Transurethral Resection of the Prostate (TURP), there are some significant differences that contribute to a cost differential between the two modalities. Given the necessity to use hypotonic irrigation fluid and the risk of fluid absorption, time is restricted with monopolar TURP. Therefore, operative times are generally less with monopolar TURP than with HoLEP, where the use of saline negates any such restrictions and allows prostates of any size to be treated [1]. When training residents surgical times tend to be longer and therefore the lack of restricttion on operative time with HoLEP over TURP is even more magnified. In addition, the use of a morcellator

\footnotetext{
*Dr. Lori Lerner works as a Consultant and Preceptor for Lumenis, Inc. There was no funding source in this study.

${ }^{\#}$ Corresponding author.
}

with HoLEP for tissue extraction adds another level of complexity to the case, which is reflected in work value assigned to HoLEP. The American Medical Association has assigned Medicare Work Relative Value Units (RVUs) of 17.29 for HoLEP, procedure code 52649, and 15.26 for TURP, procedure code 52601 [2]. Therefore, some of the difference in time spent in the operating room is offset by higher reimbursement, which varies by state and insurance carrier. Moreover, post operative hospitalizetion and acuity of care is less with HoLEP [1], which offsets the operative costs even further. All that said, variances in operative time will still impact costs, as does a difference in disposables for the two procedures. Few studies to date have attempted to directly analyze this cost differential, and none have evaluated procedures selected and performed by residents. We set out to determine the differences in dollar costs between TURP and HoLEP at VA Boston Healthcare system, a teaching 
institution with urology residents from both Boston University Medical Center and the Harvard/Longwood Program. This study was approved by the VA Boston Healthcare System Internal Review Board.

\section{Materials and Methods}

All patients undergoing TURP at VA Boston Healthcare System from January 2007-August 2010 or HoLEP from April 2008-August 2010 were identified and reviewed. Decision regarding procedure choice was at the discretion of the senior resident in consultation with an attending surgeon. Patients who carried a diagnosis of prostate cancer, had simultaneous procedures at the time of their TURP or HoLEP, had their procedure performed without a resident, or had prior prostate/urethral procedures were excluded from analysis. All HoLEP procedures were supervised by one attending surgeon (LBL), while TURP procedures were supervised by one of 5 attending surgeons. All included procedures involved a resident as primary surgeon and were considered teaching cases. Tissue retrieved at the time of surgery was placed in formalin $10 \%$ and weighed by pathology after full fixation. All costs from the day of surgery to 6 months postoperatively were captured and analyzed. Only costs directly related to operative, post-operative, and urologic follow-up were included. Costs were determined by the Department of Decision Support Services (DSS) and represent actual costs accrued at our institution, irrespective of any reimbursement. Statistical analysis was performed using Student's T-Test for continuous variables, and Fisher's exact test for categorical variables.

\section{Results}

38 patients who underwent HoLEP, and 23 who underwent TURP met inclusion criteria. Patient characteristics were compared and are presented in Table 1. The two groups were comparable as regards age and PSA. PSA was used as a surrogate for prostate size as pre-operative TRUS was not routinely performed and comprehensive information on prostate volume was not available. For those patients on finasteride (a 5 alpha reductase inhibittor), a corrected PSA was calculated by doubling measured PSA value, revealing no statistical difference between the groups. However, patients undergoing HoLEP had statistically higher American Society of Anesthesiology (ASA) classification, signifying a higher risk patient population, generally from more co-morbidities. Additionally, HoLEP patients were more likely to be continued on either aspirin or Coumadin perioperatively. Despite this, average length of hospital stay was significantly less for patients undergoing HoLEP (Table 2). Cost comparisons are represented in Table 2. Total costs were significantly higher for HoLEP at approximately $30 \%$ more than TURP (a difference of \$2518), based on increased operative costs and increased operative time. Perioperative costs were lower for HoLEP due to a decreased length of stay, however, this was not statistically significant.

Table 1. Patient demographics and characteristics.

\begin{tabular}{|c|c|c|c|c|c|c|c|c|}
\hline & $\mathrm{n}$ & $\begin{array}{l}\text { Median Age } \\
\text { (years) }\end{array}$ & $\begin{array}{l}\text { Average ASA } \\
\text { Class }\end{array}$ & $\begin{array}{l}\text { Average PSA } \\
(\mathrm{ng} / \mathrm{dl})\end{array}$ & $\begin{array}{c}\text { 5ARI } \\
(\%)\end{array}$ & $\begin{array}{l}\text { Average Corrected } \\
\text { PSA (ng/dl) }\end{array}$ & $\begin{array}{l}\text { Aspirin Continued } \\
\text { ( } \% \text { of total) }\end{array}$ & $\begin{array}{c}\text { Coumadin Continued } \\
\text { (\% of total) }\end{array}$ \\
\hline HoLEP & 38 & $\begin{array}{c}64 \\
(54-82)\end{array}$ & 3.125 & $\begin{array}{c}3.70 \\
(0.5-10.6)\end{array}$ & 55.17 & $\begin{array}{c}5.89 \\
(0.5-19.08)\end{array}$ & 15.79 & 7.89 \\
\hline TURP & 23 & $\begin{array}{c}67.93 \\
(58-83)\end{array}$ & 2.77 & $\begin{array}{c}3.47 \\
(0.56-12.5)\end{array}$ & 50.00 & $\begin{array}{c}4.61 \\
(0.56-13.96)\end{array}$ & 4.35 & 0.00 \\
\hline $\mathrm{p}$ & & 0.88 & $<0.05$ & 0.81 & 0.62 & 0.30 & & \\
\hline
\end{tabular}

Ranges displayed in parentheses.

Table 2. Operative data and costs.

\begin{tabular}{|c|c|c|c|c|c|c|}
\hline & $\begin{array}{r}\text { Mean Operative } \\
\text { (Minutes) }\end{array}$ & $\begin{array}{c}\text { Time Average Hospital Duration } \\
\text { (Days Post-Operatively) }\end{array}$ & $\begin{array}{l}\text { Tissue Weight after } \\
\text { Fixation (grams) }\end{array}$ & $\begin{array}{c}\text { Average Operative Cost } \\
\text { (USD) }\end{array}$ & $\begin{array}{c}\text { Average Peri-Operative } \\
\text { Cost }\end{array}$ & Total \\
\hline HoLEP & $\begin{array}{c}128 \\
(36-258)\end{array}$ & $\begin{array}{c}0.43 \\
(0-1)\end{array}$ & $\begin{array}{c}20.71 \\
(5-40)\end{array}$ & $\begin{array}{c}\$ 6768.14 \\
(\$ 2040-\$ 9869)\end{array}$ & $\begin{array}{c}\$ 1611.86 \\
(\$ 185-\$ 9886)\end{array}$ & $\begin{array}{c}\$ 8380.00 \\
(\$ 4368-\$ 13,536)\end{array}$ \\
\hline TURP & $\begin{array}{c}77 \\
(31-136)\end{array}$ & $\begin{array}{l}1.25 \\
(1-2)\end{array}$ & $\begin{array}{c}15.22 \\
(3.5-29)\end{array}$ & $\begin{array}{c}\$ 3853.35 \\
(\$ 1312-\$ 6409)\end{array}$ & $\begin{array}{c}\$ 2008.43 \\
(\$ 742-\$ 4572)\end{array}$ & $\begin{array}{c}\$ 5861.78 \\
(\$ 2591-\$ 9241)\end{array}$ \\
\hline p-value & $<0.05$ & $<0.05$ & $<0.05$ & $<0.05$ & 0.31 & $<0.05$ \\
\hline
\end{tabular}

Ranges displayed in parentheses. 


\section{Discussion}

It is apparent that HoLEP is more time consuming than TURP, requires more time to teach, and therefore costs more than TURP. There has been much discussion in the literature and at academic conferences regarding the learning curve of HoLEP. Indeed, there is a learning curve to TURP, as well. In fact, simulators have been created to attempt to reduce complications encountered during the learning curve and improve patient outcomes [3]. We attempted to look at how resident patient selection and the learning curve of both these procedures impacted costs, in the absence of any training experience on a simulator for either procedure.

Once HoLEP became an available option at VA, it was more often selected than TURP, as can be seen by the disparity in the numbers of patients in each group, despite the larger time frame we utilized for TURP. Given the higher ASA class in the HoLEP group, it appears that when given the option of both procedures, residents, in consultation with up to 5 attending surgeons (only one of whom performs HoLEP), preferred HoLEP over TURP in higher risk patients. This suggests that both residents and attending staff likely believed that HoLEP was a safer approach over monopolar TURP, at least for some of the patients. Certainly this was true for patients in whom anticoagulants of any kind were required during surgery. Despite the higher risk population, the HoLEP group had a decreased hospital stay and less post operative costs up to 6 months from surgery, suggesting minimal early post operative complications that required clinic and/or hospital visits, admission, or interventions, irespective of resident teaching during the case.

There is a clear time limit of TURP, regardless of how much tissue is removed and how well the resident is doing. The teaching time differences are more exaggerated in HoLEP as there is no time restriction. Therefore, the largest impact of cost differences between the two procedures in a teaching hospital is operative time, as shown by HoLEP having significantly higher operative costs than TURP (a difference of \$2915). The difference in operative time was expected and has been shown in previous publications [4-6] While more tissue is removed than with TURP, enucleating along the surgical capsule with tissue retrieval is a longer process [5], exaggerated even further when every case involves teaching residents at various levels of training. It has been shown that HoLEP has a steep learning curve and takes longer to perform, and teach, than TURP [4-6]. That said, enucleation efficiency does increase with experience and the operative times for an experienced HoLEP practitioner will be less than those reported here [7]. Despite the differences in time, however, other operative costs should not be impacted by residents or teaching (equipment, disposables, medications, etc.) and are further discussed.
The Veterans Administration splits costs into variable and fixed costs, which are similar to the designation of direct and indirect costs at other institutions. Only variable costs, which are those directly related to surgery, were included in the analysis. No pre-operative costs were included as these would be equivalent between the patient groups. Costs were captured from the moment the patient arrived the morning of their surgery through 6 months of follow-up. Therefore, costs included the admission to the preoperative day unit, surgery, disposables associated with the surgery (including the laser fiber or resection loop), postoperative care, observation and/or admission costs (if the patient was kept in hospital), and all clinic visits, procedures, and/or readmissions seconddary to surgery up to 6 months. Professional fees were included, both for the surgery and admission, which varies from a previously reported study by Goh et al. which did not include professional fees in their comparison of PVP vs. TURP in two private tertiary hospitals in Houston, Texas [8]. Their cost for TURP was $\$ 5097 \pm \$ 5003$, while ours was $\$ 5861.78 \pm \$ 2446$ standard deviation (range \$2591 - \$9241), for a difference of \$764.78. If professional fees are removed from the VA Boston costs, the two costs would be very similar. In addition, their study was in a private, non academic setting so we would expect their operative time to be less.

While holmium laser fibers can be re-used, the leadership at VA Boston has chosen to dispose of every fiber after a single use to reduce workload within the central processing unit secondary to staffing issues. Each fiber at VA Boston costs $\$ 540$ and morcellator tubing costs $\$ 36$. A resection loop is $\$ 93$. Therefore, $\$ 483$ of difference are directly accounted for by unique disposables. In institutions where the holmium fibers are re-used, there will be a cost savings. If the differences in reimbursement are also calculated, with HoLEP being reimbursed at a higher rate than TURP, the total difference between the two procedures shrinks further [2].

Comment should be made about the cost of the laser and the diathermy unit, amortization, and maintenance of both the units. Equipments purchases are made through a separate equipment budget and are not included in the daily utilization costs. Similarly, maintenance contracts fall under an overall operative room operating budget and likewise are not directly included in the individual operative costs. However, operative costs do take the operating budget into account and the costs of maintenance are "shared" by all procedures. Therefore, it is fair to say that maintenance of all operating room equipment is divided amongst all services and all procedures and treated equally.

Patient care setting and length of stay have been shown to be strong independent predictors of total cost when comparing photoselective vaporization (PVP) to TURP 
[8]. Like PVP, HoLEP can be performed in an ambulatory care surgicenter environment on an out-patient basis. In our study, length of stay was less than TURP and perioperative costs therefore trended in favor of HoLEP. Our length of stay for both procedures, however, was better than that published in other studies, irrespective of the teaching nature of all our cases.

The costs we reported are for VA Boston Healthcare System, only, one of the most expensive veterans hospitals in the country. Despite this, the $30 \%$ difference between the two procedures can likely be applied to any set of costs incurred by a patient in another region or teaching environment to determine an approximate cost differential, recognizing some of the cost saving potentials listed above and eliminating resident education from the time differential.

Reported re-operation rates for HoLEP range from $1.4 \%-4.5 \%$ up to six years $[9,10]$, and $5.6 \%-14.7 \%$ for TURP at longer durations of $8-10$ yrs [11,12]. HoLEP has been shown to result in larger amounts of resected tissue as the procedure is performed along the surgical capsule, much like an open simple prostatectomy $[6,13]$. Since all the adenoma is removed directly along the capsule, it is expected that re-operation rates for adenomatous regrowth would be lower than TURP. If TURP patients require another surgery during their lifetime, the operative costs will clearly swing in favor of HoLEP. Given the lower reoperation rates, long term costs of HoLEP are likely lower than TURP. Therefore, while the upfront operative costs of HoLEP are higher, the long term savings is likely significant.

Several comments should be made. First, our tissue weights are lower than what we expected. Clearly, the impact of the learning curve can be seen in both these procedures in the amount of tissue that was resected, with trainees removing less than what would be expected from an experienced urologist. In addition, the fixation of the specimen may have had an impact. At VA Boston, TURP and HoLEP specimens are immediately placed in formalin and not sent fresh. It has been shown that formalin reduces tissue weight by up to $25 \%$, depending on the water content of the cells making our adjusted fresh weight higher $[14,15]$. Many institutions send specimens fresh and the weights recorded are therefore higher than ours.

A second limitation is the relatively low number of cases in both groups over the study period. First, this emphasizes the impact of medical therapy on the volume of surgery for BPH, even amongst a group of residents motivated to recruit surgical patients for their own learning experience. However, we also had a large number of excluded patients as many patients were post radiation, had previous urethral and/or prostate surgery, pelvic trauma, etc. Veterans hospitals are not reflective of a typical pri- vate practice and are unable to pull patients from a wide population. Patients treated are those that are already within the system and therefore there is a limited pool of patients and minimal ability to "grow" a practice. In order to provide meaningful comparisons, it was essential to find two patient groups within this population without confounding issues. Despite the low numbers, the study was still powered enough to show meaningful differences.

Lastly, some of the HoLEP procedures included operative times up to $4 \mathrm{hrs}$. Length in these procedures was partly related to equipment issues (broken fiber, faulty morcellator, etc.) and not necessarily related to teaching and/or learning. However, these things are unique to HoLEP and are therefore part of the operative time. That said, it is important to clarify that not all the stated time in these instances was spent enucleating and morcellating.

\section{Conclusion}

It is clear that performing HoLEP at a VA hospital with residents costs more than TURP, secondary to higher operative times. We can presume that for an attending surgeon who develops experience in HoLEP, the cost difference may not be nearly as dramatic as the operative times would likely be shorter. Despite the significantly higher cost of HoLEP, reimbursement is higher for HoLEP, perioperative costs are lower and recovery time faster, even during training. Medically complex patients, including those on anticoagulation, were more likely to be selected for HoLEP, as reflected by higher ASA scores, but the costs for treating these patients was no different. So while teaching and performing HoLEP may cost more from a surgical perspective, when all factors are considered, including reimbursement, this difference is relatively minimal. Given the faster recovery and broader patient population that can undergo surgical therapy for their symptomatic bladder outlet obstruction with HoLEP, this expense may be well worth the minor and short term cost difference.

\section{REFERENCES}

[1] L. B. Lerner and M. D. Tyson, "Holmium Laser Applications of the Prostate," Urologic Clinics of North America, Vol. 36, No. 4, 2009, pp. 485-495. doi:10.1016/j.ucl.2009.07.005

[2] http://www.ama-assn.org/OCM/CPTRelativeValueUnit Accessed 18 September 2011.

[3] S. J. Hudak, C. L. Landt, J. Hernandez and D. W. Soderdahl, "External Validation of a Virtual Reality Transurethral Resection of the Prostate Simulator," The Journal of Urology, Vol. 184, No. 5, 2010, pp. 2018-2022. doi:10.1016/j.juro.2010.06.141 
[4] R. M. Kuntz, S. Ahyai, K. Lehrich and A. Fayad, "Transurethral Holmium Laser Enucleation of the Prostate versus Transurethral Electrocautery Resection of the Prostate: A Randomized Prospective Trial in 200 Patients," The Journal of Urology, Vol. 172, No. 3, 2004, pp. 1012-1016. doi:10.1097/01.ju.0000136218.11998.9e

[5] R. M. Mavuduru, A. K. Mandal, S. K. Singh, N. Acharya, M. Agarwal, S. Garg and S. Kumar, "Comparison of HoLEP and TURP in Terms of Efficacy in the Early Postoperative Period and Perioperative Morbidity," Urologia Internationalis, Vol. 82, No. 2, 2009, pp. 130-135. doi: $10.1159 / 000200786$

[6] A. H. Tan, P. J. Gilling, K. M. Kennett, C. Frampton, A. M. Westenberg and M. R. Fraundorfer, "A Randomized Trial Comparing Holmium Laser Enucleation of the Prostate with Transurethral Resection of the Prostate for the Treatment of Bladder Outlet Obstruction Secondary to Benign Prostatic Hyperplasia in Large Glands (40 to 200 Grams)," The Journal of Urology, Vol. 170, No. 4, 2003, pp. 1270-1274. doi:10.1097/01.ju.0000086948.55973.00

[7] H. N. Shah, A. P. Mahajan, H. S. Sodha, S. Hegde, P. D. Mohile and M. B. Bansal, "Prospective Evaluation of the Learning Curve for Holmium Laser Enucleation of the Prostate," The Journal of Urology, Vol. 177, No. 4, 2007, pp. 1468-1474. doi:10.1016/j.juro.2006.11.091

[8] A. C. Goh and R. R. Gonzalez, "Photoselective Laser Vaporization Prostatectomy versus Transurethral Prostate Resection: A Cost Analysis," The Journal of Urology, Vol. 183, No. 4, 2010, pp. 1469-1473. doi:10.1016/j.juro.2009.12.020

[9] P. J. Gilling, T. F. Aho, C. M. Frampton, C. J. King and M. R. Fraundorfer, "Holmium Laser Enucleation of the Prostate: Results at 6 Years," European Urology, Vol. 53,
No. 4, 2008, pp. 744-749. doi:10.1016/j.eururo.2007.04.052

[10] E. A. Elzayat and M. M. Elhilali, "Holmium laser Enucleation of the Prostate (HoLEP): Long-Term Results, Reoperation Rate, and Possible Impact of the Learning Curve," European Urology, Vol. 52, No. 5, 2007, pp. 1465-1471. doi:10.1016/j.eururo.2007.04.074

[11] O. Reich, C. Gratzke and C. G. Stief, "Techniques and Long-Term Results of Surgical Procedures for BPH," European Urology, Vol. 49, No. 6, 2000, pp. 970-978. doi:10.1016/j.eururo.2005.12.072

[12] G. L. Lu-Yao, M. J. Barry, C. H. Chang, J. H. Wasson and J. E. Wennberg, "Transurethral Resection of the Prostate among Medicare Beneficiaries in the United States: Time Trends and Outcomes. Prostate Patient Outcomes Research Team (PORT)," Urology, Vol. 44, No. 5, 1774, pp. 692-698.

[13] R. M. Kuntz, K. Lehrich and S. A. Ahyai, "Holmium Laser Enucleation of the Prostate versus Open Prostatectomy for Prostates Greater than 100 Grams: 5-Year Follow-Up Results of a Randomised Clinical Trial," European Urology, Vol. 53, No. 1, 2008, pp. 160-166. doi:10.1016/j.eururo.2007.08.036

[14] R. C. Augusteyn, G. Vrensen and B. Willekens, "The Effect of Paraformaldehyde Fixation and PBS Storage on the Water Content of the Human Lens," Molecular Vision, Vol. 14, 2008, pp. 90-94.

[15] S. Jonmarker, A. Valdman, A. Lindberg, M. Hellström and L. Egevad, "Tissue Shrinkage after Fixation with Formalin Injection of Prostatectomy Specimens," Virchows Archiv, Vol. 449, No. 3, 2006, pp. 297-301. doi:10.1007/s00428-006-0259-5 